ARTICLE

\title{
Adapting the psychiatric assessment for primary care
}

\author{
J Parker, MB BCh, FCPsych (SA) \\ OPD, Outreach and Medium Term Services, Lentegeur Hospital, Division of Public Mental Health, Department of Psychiatry and Mental Health, \\ University of Cape Town, South Africa
}

Corresponding author: J Parker (john.parker@westerncape.gov.za)

S Afr Med J 2014;104(1):75. DOI:10.7196/SAMJ.7731

口it?

A particular challenge in primary healthcare is that the time available for consultations is extremely limited owing to high workloads. This can be particularly difficult in the detection and management of mental health problems. ${ }^{[1]}$ Traditionally, the mental health assessment has been taught as a very comprehensive psychiatric history and examination, which can take as long as an hour. While the value of thorough assessment cannot be denied, such an imperative becomes self-defeating if it discourages any assessment at all, or results in one that misses the most critical information.

An important step, therefore, in developing mental healthcare at the primary level, is to establish a means of assessment that allows for a short consultation, yet also ensures that the most vital information is not missed. This can be achieved if there is a solid understanding of the purpose of the comprehensive psychiatric history and examination, and a knowledge base and skill set is developed that is congruent with this purpose. Fortunately, such knowledge, understanding and skills generally involve an elaboration and enhancement of existing areas of ability rather than something unique.

This requires a focus on the following key areas:

- identification of mental illness

- establishment of rapport in difficult situations

- engagement and involvement of others who may be affected by the illness

- use of the assessment as a therapeutic tool

- understanding of its nature as a continuous work in progress

- identification and management of risk

- development of hypotheses in the course of the interview and the ability to adjust the interview to allow for the testing of these

- use of a syndromic approach to do so.

\section{Identifying the presence of mental \\ illness}

The ability to identify the presence of mental illness is critical. This is because, for a number of reasons, many people with a mental illness will either not initially be willing to identify within themselves the possibility of mental illness, or will not be aware that their symptoms are due to a mental illness. To make this easier, it is useful to be aware of when to suspect mental illness and how to screen for it.

When to consider mental illness:

- when you are aware of the existence of marital, sexual or relationship problems

- when there are complaints attributed to supernatural causes

- when there have been complaints of family violence, any form of abuse or emotional or severe physical trauma

- when you are aware that the person has life problems, such as unemployment or the death of a close friend or relative
- when you are aware that the person is suffering from a chronic and severe physical illness and particularly if the diagnosis is associated with stigma

- when there are many physical complaints (especially more than three) that do not fit with any recognised pattern of physical illness

- when the person has physical complaints that fail to respond to the appropriate treatment

- when there is a personal or family history of mental illness.

\section{Screening for mental illness}

Perhaps the most useful screening questions to ask are those set out by Vikram Patel ${ }^{[2]}$ in his book, Where There is No Psychiatrist, as the 'golden questions':

- Do you have any problems sleeping at night?

- Have you been feeling as if you have lost interest in your usual activities?

- Have you been feeling sad or unhappy recently?

- Have you been feeling scared or frightened of anything?

- Have you been worried about drinking too much alcohol/using drugs recently?

- How much money and time have you been spending on alcohol/ drugs recently?

If any of the answers to these questions raise your suspicions, a more detailed assessment is required.

\section{Establishing and maintaining rapport}

The most important initial goal in any psychiatric interview is to establish and develop a trusting relationship with the patient. Unless this is done sufficiently well, the information obtained will be of uncertain validity, and the opportunity to effect any improvement at all will be severely compromised. It is crucial to understand that just about all psychiatric disorders affect the individual in a manner that is highly personal and that to accept that one may be mentally ill, is extremely difficult. To do this and then to take the further steps that the treatment may require, demand a singular determination and courage.

Areas that require particular attention relate to one's personal conduct and how one approaches difficult situations. In this regard, it is important to acknowledge and set aside personal feelings that may interfere with one's ability to establish a therapeutic relationship. In dealing with a mentally ill person, these may include feelings of fear, amusement, disgust or embarrassment and even anger about complaints that do not seem to constitute a 'real illness'. Ignoring the presence of mental illness usually results in it getting worse and leads to more work for one in the long run, but more importantly perhaps, learning to accept and manage one's own emotions can result in a sense of personal growth that is possibly one of the greatest gifts that our profession has to offer. A useful 
strategy to assist in managing one's emotions involves allocating oneself a minute or two between each patient, just to re-establish a sense of who one is and to remind oneself of one's primary purpose in this work.

\section{Involving others}

While confidentiality is vital, it must be counterbalanced with a number of factors, including:

- the importance of risk assessment

- the particular problem of insight in mental illness

- an understanding of the complexity of mental health problems and of the importance of context in diagnosis.

Therefore, the importance of obtaining collateral information cannot be overemphasised. This should be made clear early on in the course of an assessment with a brief discussion that establishes both the importance of confidentiality with regard to sensitive matters but also its limits in terms of legal and safety imperatives. One should seek permission to obtain information from others and to motivate for the enlistment of significant others in the healing process. Generally, and particularly with children and adolescents, the patient should be allowed to speak first, in private, before hearing from others. Exceptions to this rule, however, should be considered in cases where there is very poor insight or severely disturbed behaviour. It may then be strategic to obtain information about the nature of the problem first, before discussing it with the patient, to save time. In these instances, one should first meet briefly with everyone involved to remind them that the clinician's primary responsibility is towards the patient and to give the reassurance that each party will have an opportunity to be heard.

\section{The assessment as therapy and as a work in progress}

As mentioned above, a particular difficulty in managing mental illness relates to the development of insight or an understanding that one is suffering from an illness and of the need to take what are often difficult steps to deal with it. As such, the initial assessment plays a crucial role in the initiation of a collaborative process of problem identification and of strategies to deal with it. Most mental health disorders are complex and multidimensional in all areas of aetiology, presentation and management. Thus, any approach that involves no more than coming to a categorical, one-line diagnosis and the prescription of a medication is likely to be highly simplistic and doomed to failure. ${ }^{[3]}$ Instead of trying to pretend that this is possible, the practitioner should rather aim to make this clear early in the assessment and to set out the consultation as a work in progress, gradually developing a better understanding of a problem and of the variety of resources that may be available to aid in recovery. It is useful to explain that most effective psychiatric medication involves fairly long-term use - and therefore commitment - and that most treatments take some time to take effect. So, for both patient and practitioner, it is particularly important that treatment decisions are not rushed or based on insufficient information. Additionally, the process of uncovering information and framing it in terms that enable a better understanding of the problem and its management is in itself therapeutic: it places the presenting crisis into a broader context that provides relief and empowers the patient and others affected by it to consider alternative perspectives that may lead to solutions

Having and sharing this understanding ensures that any time that is available, however short, can be put to good use and that the problem is not avoided. It also militates against the overly simplistic understanding of complex issues and the danger of falling for the idea of a 'quick fix'.

\section{Identifying and managing risk}

Having established that a full assessment is unlikely to be completed at the first interview, it becomes imperative that one is clear about what information must be obtained and what kind of management may need to be prioritised. A hierarchy of risks needs to be considered, beginning with the risk of danger to self and the risk of danger to others, and with those risks that result directly from harmful acts assuming a priority over those that may occur as a result of indirect harm or neglect. The latter would include cases where the danger involves a person's financial wellbeing or reputation and a consideration of any dangers relating to delays in providing treatment for the presenting illness, particularly where this may be due to delirium. In the case of minors, or of other vulnerable individuals with impaired or reduced decision-making capacity, another important possibility is whether they are being exposed to any form of abuse, or the withholding of their rights. Finally, it is also important to consider the person's sense of safety, the level of personal distress that they and those around them may be experiencing, and how well they are able to function on personal, social and occupational levels. Where any level of risk has been identified, this must be recorded and acted upon. Factors which must be determined and noted are:

- details of the risk factor

- seriousness of the risk

- specificity of the risk to an individual

- how immediate the risk is

- what interventions are required to reduce the risk and what steps have been taken. ${ }^{[4]}$

\section{Hypothesis formation and a simplified approach to diagnosis}

It is important to bear in mind that, in psychiatry, achieving a definitive diagnosis after the first interview is seldom either possible or desirable. It is extremely rare that all the necessary information will immediately become available, so it is generally unwise and may even be dangerous to draw conclusions too rapidly. This is, however, often easier said than done as the high levels of anxiety associated with many presentations can place significant pressure on the clinician to suggest answers that are definitive. It may be helpful to declare up front that, because we are such complex beings, it will take some time to develop a full understanding of the problem and its solutions, but this process does not need to be completed for the healing to begin. Indeed, in many cases, the process of discovery will, in itself, begin to bring relief. So the key is to demonstrate confidence and a clear direction in dealing with the uncertainty that will arise, rather than going for the fool's gold of a hasty conclusion.

In this regard, it is therefore crucial to have a diagnostic framework in place that allows one to work logically and methodically towards a helpful formulation. A useful way of doing this is to think in terms of broad priority syndromes rather than individual diagnoses. One can then organise one's history taking to enable rapid identification of the most likely syndrome. Once this has been identified, it becomes possible to pursue investigations that are specific to that particular syndrome and its profile of risk factors, aetiological agents and management strategies. An outline of such an approach is described in 'A broad diagnostic framework to simplify the approach to mental disorders in primary care. ${ }^{[5]}$ 


\section{Summary}

- The traditional form of psychiatric assessment needs to be shortened to facilitate its use at the primary care level.

- This can be done if there is a good understanding of the aims and purposes of a mental health assessment and its nature as a work in progress.

- Because many people with mental illness struggle to identify the preceding, it is important to know when to suspect it and what questions to ask.

- Developing and maintaining rapport is vital for a successful assessment, but doing so simply requires attention to skills that should be basic for any practitioner.

- Collateral information is vital in many instances.

- It is seldom possible or desirable to reach a definitive diagnosis and treatment plan at the first assessment, so this should be seen as the beginning of a collaborative process of investigation that, in and of itself, has therapeutic value.
- It is vital to identify what steps must be taken urgently at the first assessment, so a careful risk assessment is crucial. This requires a clear understanding of the hierarchy of risks that may be present.

- A diagnostic framework of broad syndromal categories will then provide a basis to direct further enquiry.

\section{References}

1. Thielke S, Vannoy S, Unutzer J. Integrating mental health and primary care. Prim Care Clin Office Pract 2007;34:571-592. [http://dx.doi.org/10.1016/j.pop.2007.05.007]

2. Patel V. Where There is No Psychiatrist. A Mental Health Care Manual. UK: Gaskell (Royal College of Psychiatrists), 2003.

3. Bakker PR, Wichers M, Van Harten PN, et al. Novel directions for psychiatric diagnosis: From psychopathology to motor functioning to monitoring technology. Epidemiology and Psychiatric Psychopathology to motor functioning to monitoring technology.

4. McDowell AK, Lineberry TW, Bostwick JM. Practical suicide risk-management for the busy primary care physician. Mayo Clin Proc 2011;86(8):792-800. [http://dx.doi.org/ 104065 mcp.2011.0076]

5. Parker J. A broad diagnostic framework to simplify the approach to mental disorders in primary care. S Afr Med J 2014;104(1):69-71. [http://dx.doi.org/10.7196/SAMJ.7717] 